\title{
Imaging coronal magnetic-field reconnection in a solar flare
}

\author{
Yang Su ${ }^{1 \star}$, Astrid M. Veronig', Gordon D. Holman ${ }^{2}$, Brian R. Dennis ${ }^{2}$, Tongjiang Wang ${ }^{2,3}$, \\ Manuela Temmer ${ }^{1}$ and Weiqun Gan ${ }^{4}$
}

\begin{abstract}
Magnetic-field reconnection is believed to play a fundamental role in magnetized plasma systems throughout the Universe', including planetary magnetospheres, magnetars and accretion disks around black holes. This letter presents extreme ultraviolet and X-ray observations of a solar flare showing magnetic reconnection with a level of clarity not previously achieved. The multi-wavelength extreme ultraviolet observations from SDO/AIA show inflowing cool loops and newly formed, outflowing hot loops, as predicted. RHESSI X-ray spectra and images simultaneously show the appearance of plasma heated to $>10 \mathrm{MK}$ at the expected locations. These two data sets provide solid visual evidence of magnetic reconnection producing a solar flare, validating the basic physical mechanism of popular flare models. However, new features are also observed that need to be included in reconnection and flare studies, such as three-dimensional non-uniform, non-steady and asymmetric evolution.
\end{abstract}

The early concept of magnetic reconnection was proposed in the 1940s (ref. 1) to explain energy release in solar flares, the most powerful explosive phenomena in the solar system. The reconnection process reconfigures the field topology and converts magnetic energy to thermal energy, mass motions and particle acceleration. The theories and related numerical simulations, especially threedimensional modelling, are still subjects of extensive research to obtain a full understanding of the process under different conditions. Meanwhile, observational studies have made progress in finding evidence of reconnection and deriving its physical properties to constrain and improve the theories.

In situ measurements of the magnetic field, plasma parameters and particle distributions have shown the existence of magnetic reconnection in laboratory plasmas ${ }^{2,3}$, fusion facilities and magnetospheres of planets ${ }^{4,5}$. Such in situ measurements are still not possible in the extremely hot solar atmosphere. Instead, observations are obtained through remote sensing of emissions across the entire electromagnetic spectrum from radio to X-rays and gamma rays. However, in the corona ${ }^{6}$ the magnetic field pressure dominates the plasma pressure (low plasma beta) and the magnetic flux is frozen into the highly conductive plasma. As a result, the emitting plasma trapped in coronal loops outlines the geometry of the magnetic field and their structural changes reflect the changes of the field connectivity (in general). Considerable pieces of evidence for features probably linked to reconnection in solar flares ${ }^{7,8}$ and coronal mass ejections ${ }^{9}$ (CMEs) have been obtained so far. These include signatures of plasma inflow/outflow ${ }^{10-14}$, hot cusp structures $^{15}$, current sheets ${ }^{16-18}$, fast-mode standing shocks ${ }^{19}$ and plasmoid ejection ${ }^{20}$. However, most evidence has been indirect and fragmented. Detailed observations of the complete picture are still missing owing to the highly dynamic flare/CME process and limited observational capabilities.

The launch of the Solar Dynamic Observatory ${ }^{21}$ (SDO) in 2010 significantly improved this situation. In particular, the Atmospheric Imaging Assembly ${ }^{22}$ (AIA) has enabled continuous imaging of the full Sun in ten extreme ultraviolet (EUV), ultraviolet and visible channels, with a spatial resolution of $\sim 0.6 \operatorname{arcsec}$ and a cadence of $12 \mathrm{~s}$. Simultaneously, the Ramaty High Energy Solar Spectroscopic Imager ${ }^{23}$ (RHESSI) is continuing to provide X-ray imaging and spectroscopic diagnostics of the heated plasma and accelerated electrons. The flare of interest was observed close to the southeast limb of the solar disk on 17 August 2011, but no CME was detected. The peak soft X-ray flux (1-8 $\AA$ ) recorded by the X-ray monitor on a Geostationary Operational Environmental Satellite (GOES) was $2.3 \times 10^{-6} \mathrm{~W} \mathrm{~m}^{-2}$, implying a modest flare magnitude of C2.3. The favourable timing, intensity, position and orientation allowed us to observe the most complete evolution yet obtained of magnetic reconfiguration and energy release in an interacting coronal loop arcade.

The clearest visual evidence of the reconnection process comes from the synchronous imaging of cool inflow loops (Fig. 1a,b) and hot outflow loops (Fig. 1c and see also Supplementary Movies $1-3)$. From $\sim 04: 05$ ut to 04:28 ut, discrete coronal loops with temperatures from $\sim 0.05$ to $2 \mathrm{MK}$ merged and disappeared near a central plane (the red line in Fig. 1b-c), the same location where a hot X-shaped structure $(\sim 10 \mathrm{MK}$, first image in Fig. $1 \mathrm{c})$ gradually formed by $\sim 04: 10$ ut. The two cusps above and below the X-type neutral point began to separate to form a $\mathrm{V}$-inverted $\mathrm{V}$ structure at $\sim 04: 15$ uT (second image in Fig. 1c). Newly formed hot loops $(\sim 10 \mathrm{MK})$ then appeared in two separated groups. Both flow away from the reconnection region (Fig. 1c), presumably owing to magnetic tension forces.

The bright flare regions on the surface (observed at AIA 1,600 , see Fig. $2 \mathrm{~b}$ and Supplementary Fig. S1) are the footpoints of coronal loops heated by thermal conduction and/or non-thermal electrons. As a result of the reconnection, the magnetic topology probably changed from an arcade of loops connecting footpoints $\mathrm{A}-\mathrm{B}$ in the southern end and $\mathrm{C}-\mathrm{D}$ in the northern end to two vertically separated new sets of loops (Fig. 2b). One group connects the lower cusp to regions $\mathrm{A}$ and $\mathrm{C}$. This relaxes downward onto the flare arcade. The other group probably connected the higher cusp to the regions $\mathrm{B}$ and $\mathrm{D}$. This expands outward, contributing to the loops (magnetic flux rope) building above the arcade.

\footnotetext{
${ }^{1}$ Kanzelhöhe Observatory-IGAM, Institute of Physics, University of Graz, Universitaetsplatz 5/II, 8010 Graz, Austria, ${ }^{2}$ Solar Physics Laboratory (Code 671), Heliophysics Science Division, NASA Goddard Space Flight Center, Greenbelt, Maryland 20771, USA, ${ }^{3}$ Department of Physics, the Catholic University of America, Washington DC 20064, USA, ${ }^{4}$ Key Laboratory of Dark Matter and Space Astronomy, Purple Mountain Observatory, Chinese Academy of Sciences, Nanjing 210008, China. *e-mail: yang.su@uni-graz.at
} 


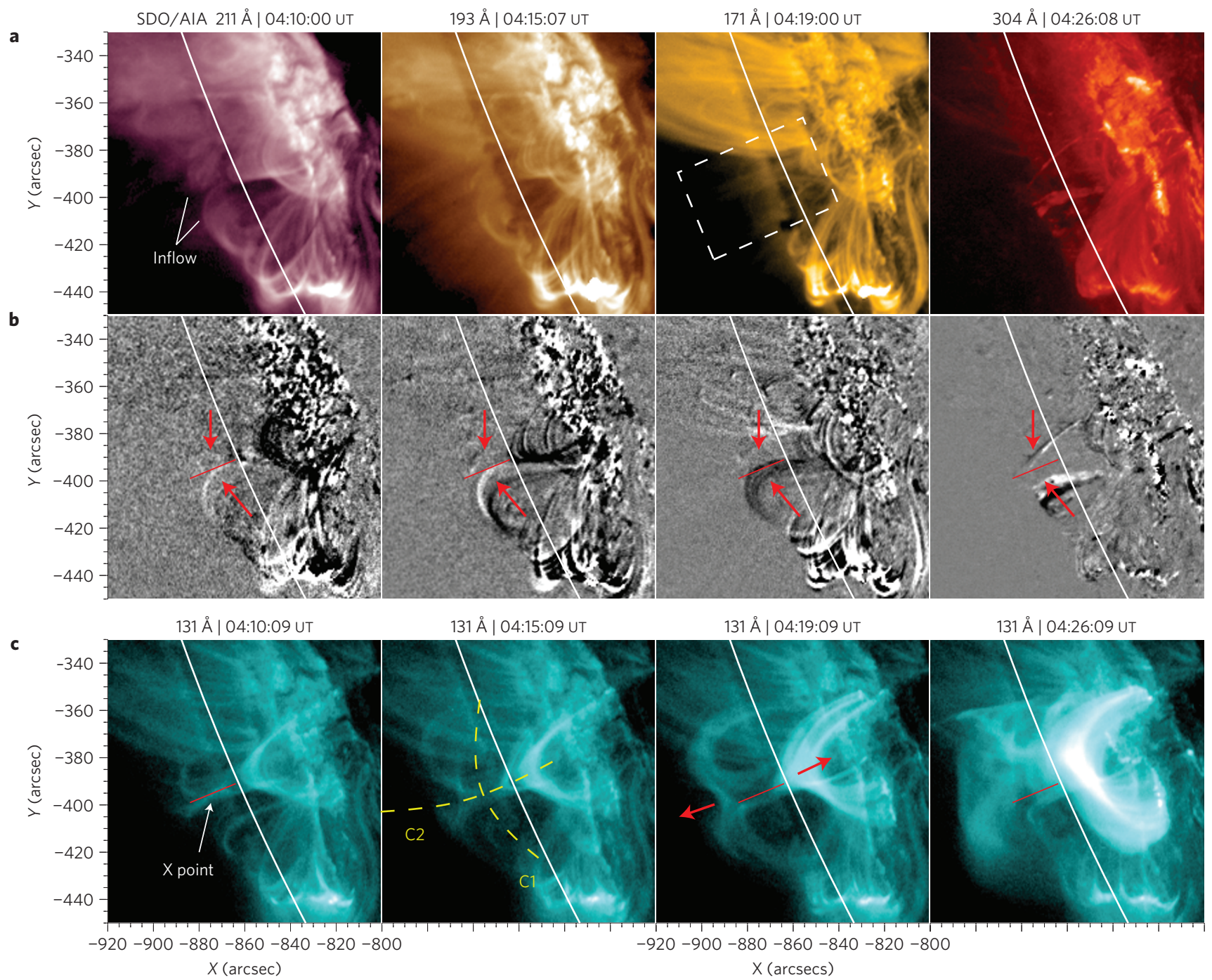

Figure 1 | SDO/AIA overview of the reconnection process observed in EUV. These false-colour images are taken from Supplementary Movies 1-3 at different times during the flare on 17 August 2011. They show cool inflow loops merging horizontally (north/south) and hotter outflow loops separating vertically (east/west). The white curved line in each frame marks the visible edge (horizon) of the solar disk. The $X$ and $Y$ coordinates of each image are in arc seconds $(1 \operatorname{arcsec} \approx 735 \mathrm{~km})$ from the disk centre. a, AIA images at $211,193,171$ and $304 \AA(1 \AA=0.1 \mathrm{~nm})$ show inflowing loops with temperatures between $\sim 0.05$ and $2 \mathrm{MK}$. The white rectangle in the third image indicates the field of view for Fig. 4. $\mathbf{b}$, The same as the images shown in $\mathbf{a}$ after subtracting images taken one minute earlier. These difference images show moved/brightened inflow loops in white relative to their original positions/intensities in black. The red line in each frame marks the initial location of the $\mathrm{X}$ structure where the inflow loops seemed to merge and disappear; the red arrows show the inflow directions. c, AIA images at $131 \AA$ showing plasma structures heated to $\sim 10$ MK. The yellow dashed curves marked $\mathrm{C} 1$ and $\mathrm{C} 2$ are used to derive the inflow and outflow profiles shown in Fig. 3. The two red arrows in the third image show the outflow directions of the hot, separating loops.

X-ray emissions from the heated plasma confirm the energy release from a reconnection process in both timing and location. The RHESSI X-ray flux began to increase at 04:06:40 UT, close to the time when the EUV inflow loops visibly started moving together (Fig. 3d). The GOES 1-8 A flux started to decrease after $\sim 04: 29$ ut, the time when the last inflow loops (Fig. 3d) were seen disappearing. On the other hand, X-ray images and spectra show heated plasma (up to $17 \mathrm{MK}$, Fig. 2c) in the reconnected flare loops. The RHESSI coronal source in the $4-10 \mathrm{keV}$ energy range (first and third images of Fig. 2a) indicates the presence of plasma at $>6 \mathrm{MK}$ at $/$ near the reconnection site. After 04:19 UT, a high coronal source appeared in the $10-20 \mathrm{keV}$ images above the higher, relaxing cusp (Fig. 2a and Fig. 4b). This source and the extended source above the flare loops are known as double coronal X-ray sources, first observed in RHESSI images alone for a different flare ${ }^{16}$. Unlike the famous single Masuda source ${ }^{19}$, which may be a signature of fast-mode standing shocks below the reconnection site, double coronal sources are thought to be related to heating in both the upper and lower outflow regions. With the support from the EUV images presented here, the double coronal sources become strong evidence for plasma heating following a reconnection process.

The formation of either a single Masuda source or double corona sources remains an open question. In particular, the higher coronal source has been rarely detected and discussed ${ }^{24}$. The absence of a clear power-law bremsstrahlung component in the RHESSI spectra may indicate a negligible contribution of heating from non-thermal electrons in this case. One mechanism that could explain the double coronal sources is the slow mode magnetosonic shocks during flux retraction, which cause heating and density enhancements in the contracting loops ejected from the reconnection site ${ }^{25}$.

To quantitatively investigate the inflow and outflow motions evident in the EUV movies, we defined two curves labelled $\mathrm{C} 1$ and 
a

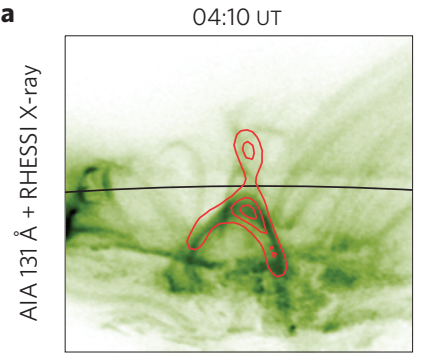

b

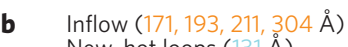

New, hot loops (131 A )

Flare ribbon $(1,600 \AA)$

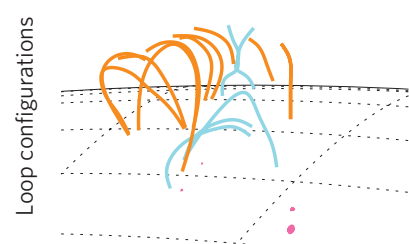

c

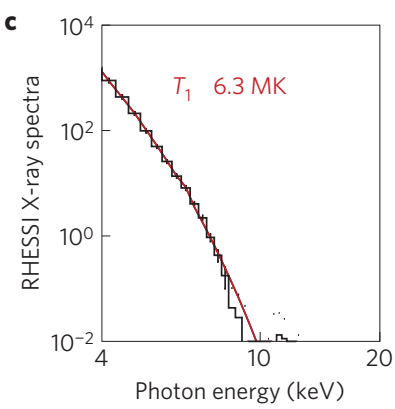

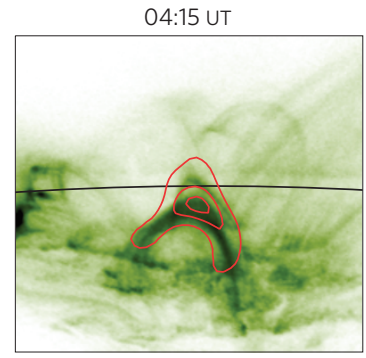
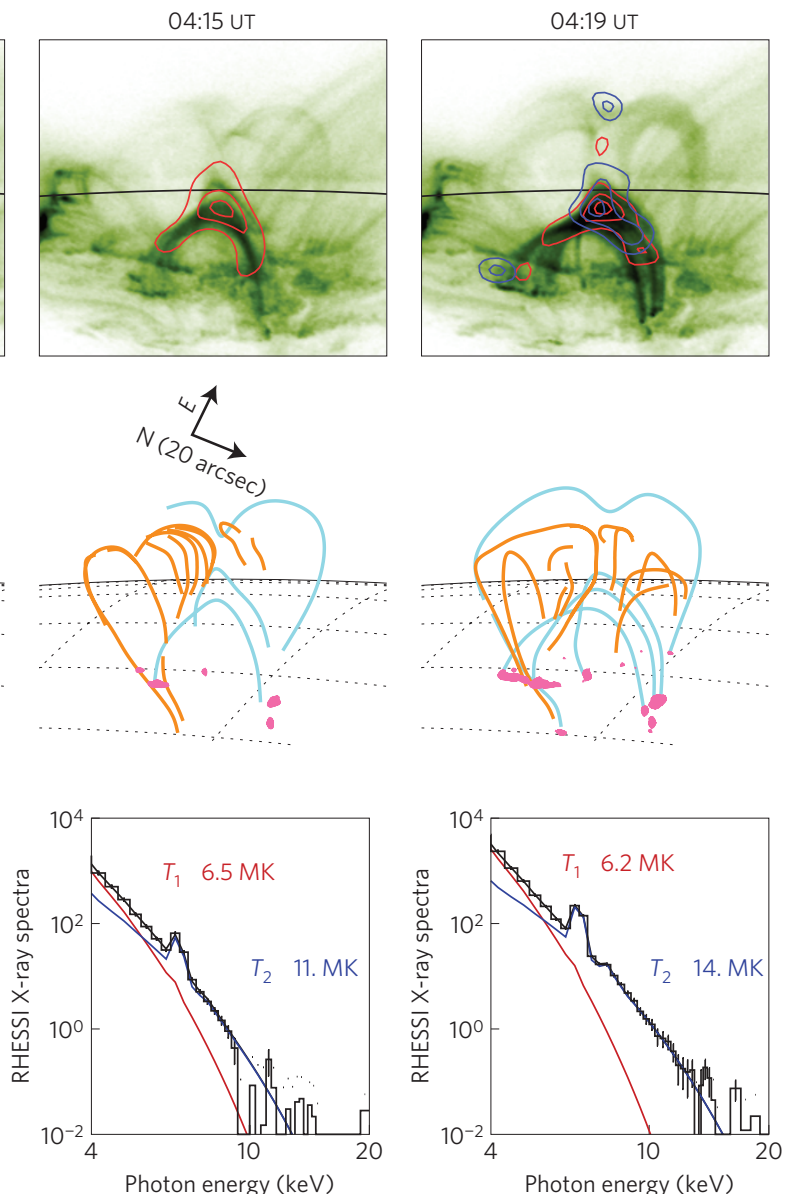
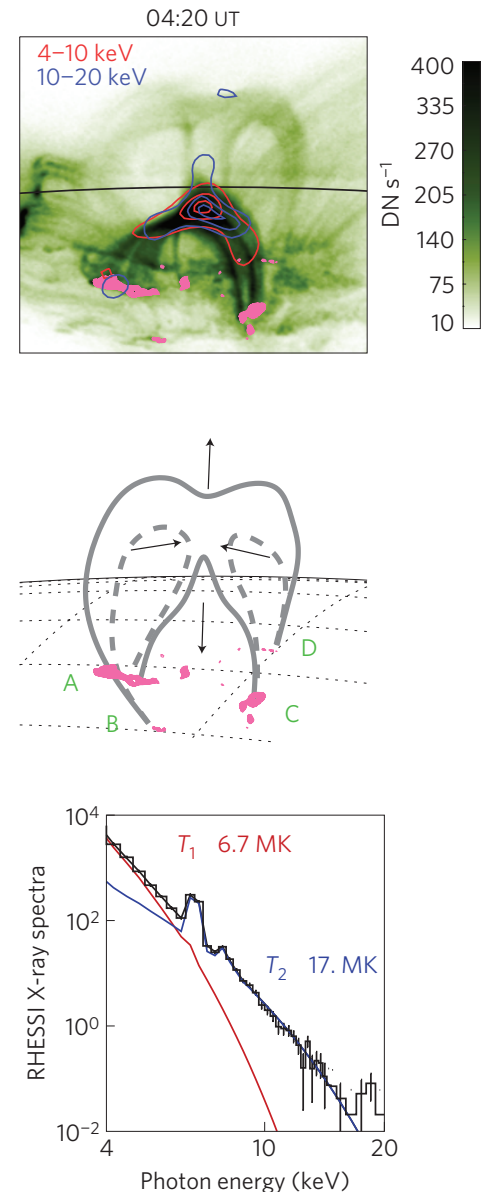

Figure 2 | The reconnection and energy release imaged by SDO/AIA in EUV and RHESSI in X-rays. The combination of data from different wavelengths shows the entire sequence of events expected for reconnection. a, AIA $131 \AA$ images, taken from Supplementary Movie 4 , show the formation of the new hot loops. The images are rotated clockwise by $114^{\circ}$ to orient the solar limb (black line) in the horizontal direction. RHESSI X-ray fluxes ${ }^{29}$ are shown as red (blue) contours in the 4-10 (10-20) keV bands at 10, 40 and $80 \%$ of the maximum in each image. Bright flare regions on the surface observed at AIA $1,600 \AA$ are superposed in pink in the fourth image. $\mathbf{b}$, The evolution of loop configurations. Orange lines show the major inflow loops derived from AIA images at 171, 193, 211 and $304 \AA$; cyan lines show the hot loops and cusps in the AIA $131 \AA$ images. Main flare regions (pink) are marked by A, B, C and D (see Supplementary Fig. S1). The dashed grey lines in the fourth image illustrate the inflowing cool loops; the solid grey lines illustrate the newly formed

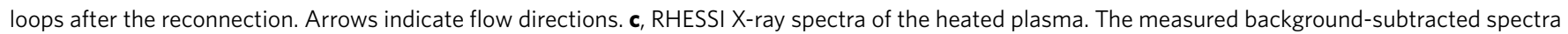

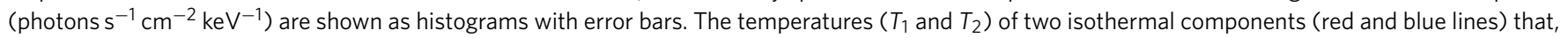
summed together (black lines), give the best fit to the data are shown for each spectrum.

C2 in Fig. 1c. The resulting time-distance plots for C1 (Fig. 3a) show coronal slices of loops that moved inward (towards the central line) from both sides and disappeared. The apparent inflow velocities increased from $\sim 10$ to over $50 \mathrm{~km} \mathrm{~s}^{-1}$ as the loops approached the point of disappearance. The final inflow velocities range from $\sim 20$ to $\sim 70 \mathrm{~km} \mathrm{~s}^{-1}$. The outflows are evident in the time-distance plots made for C2 from the $131 \AA$ images (Fig. $3 \mathrm{~b}$ ). Signatures of some superposed fast-moving structures can be seen from 04:08 to 04:22 ut (Fig. 3b and solid cyan lines in Fig. 3d) along the paths of the two separating cusps (the two dashed cyan lines in Fig. 3d). We suggest that these structures are the new loops ejected from the reconnection region, and the two cusps are the subsequently heated thermal sources. The initial outflow velocities $\left(V_{\text {out }}\right)$ range from $\sim 90$ to $\sim 440 \mathrm{~km} \mathrm{~s}^{-1}$. If we neglect the projection effect and assume ${ }^{14}$ the measured outflow velocity is approximately the local Alfvén velocity $\left(V_{\mathrm{A}}\right)$, we estimate that the reconnection rate, $M_{\mathrm{A}}=V_{\text {in }} / V_{\mathrm{A}} \approx V_{\text {in }} / V_{\text {out }}$, varies from $\sim 0.05$ to 0.5 (Fig. 3e).

The event also revealed other new features of inflows. Some inflow loops originated far from the reconnection site (up to $29,000 \mathrm{~km}$ ). The inflow loops, visible in different AIA channels, covered a wide range of temperatures from $\sim 0.05$ to $2 \mathrm{MK}$. This means that the plasma across the inflow field had different physical states. Compared with the steady, uniform case, the variable inflowing plasma could result in a variable heating rate and may help explain spikes observed in hard X-ray light curves during the impulsive phase of most flares. The highly curved inflow loops expanded as they approached the reconnection site. Detailed flow velocity maps (Fig. 4b) derived from the Fourier local correlation tracking ${ }^{26}$ method quantify this expansion. The diverging inflows, together with the signature of piling-up loops (white arrows in Fig. 3 and Supplementary Fig. S2) and the high reconnection rates, are suggestive of flux-pile-up reconnection ${ }^{1}$ rather than the classic Petschek-like reconnection ${ }^{27}$. The inflows were apparently asymmetric, assuming that inflow velocity vectors in both inflow regions are in the same plane. For example, the apparent inflow velocities at 04:20-21 UT were $\sim 50 \mathrm{~km} \mathrm{~s}^{-1}$ from the south and $\sim 20 \mathrm{~km} \mathrm{~s}^{-1}$ from the north. As the reconnection process consumes the same amount of opposite magnetic fluxes from the two inflow regions, one would expect that a weaker field requires a faster inflow speed. Then the region to the south would thus have a weaker field.

The inflow of magnetic loops is not a feature of the standard flare arcade reconnection model, in which loops within a linear 

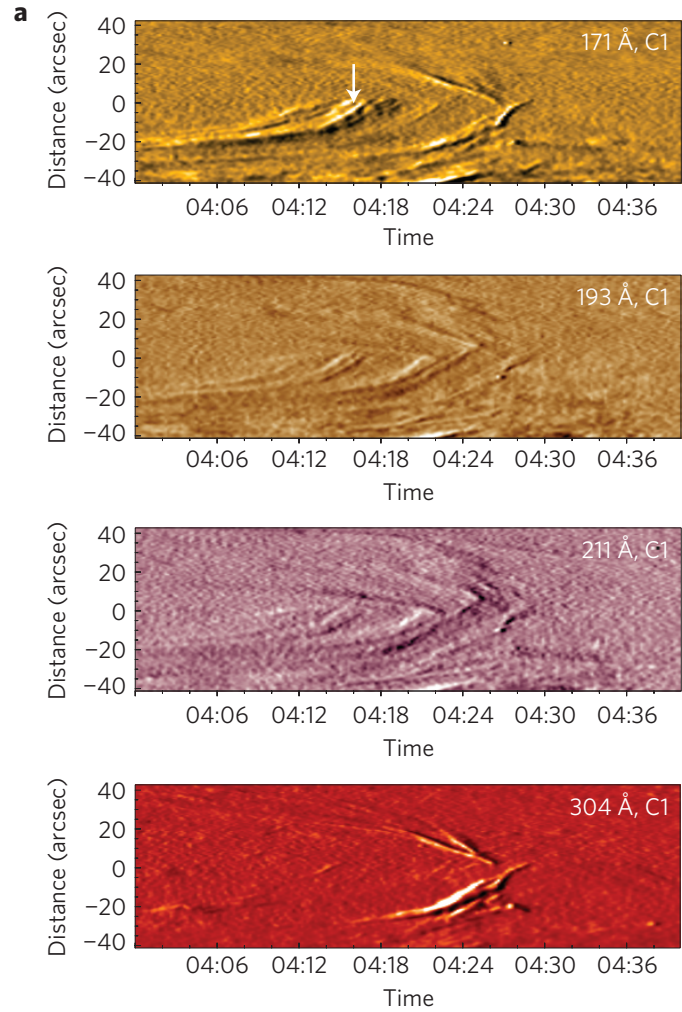

b

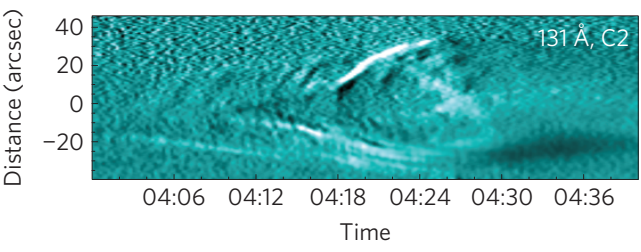

c
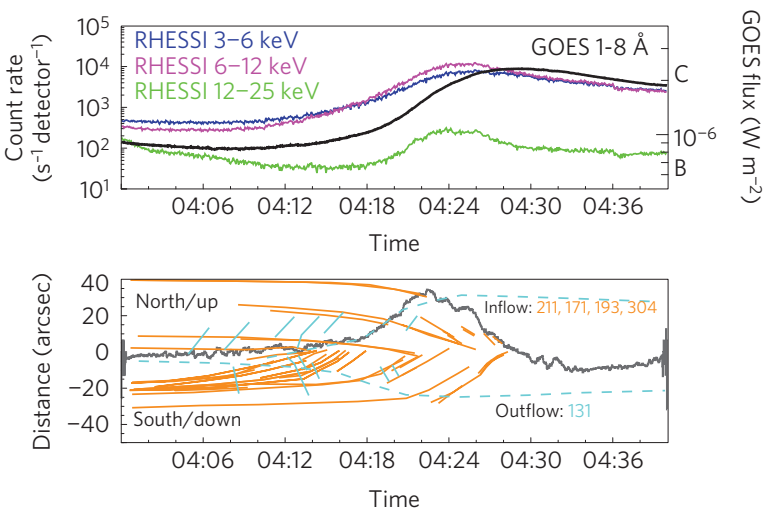

e

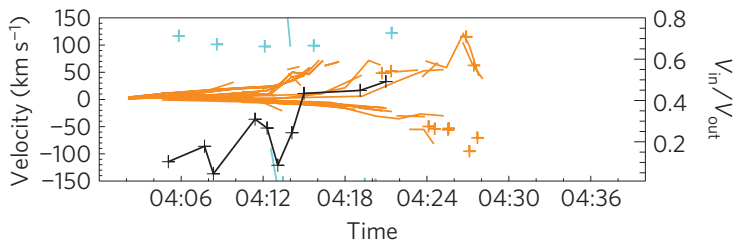

Figure 3 | Time profiles of plasma inflow, plasma outflow and X-ray flux. a, Time-distance plots (stack plots) showing (from the top) the time history of the intensity along curve $\mathrm{C} 1$ (the second image of Fig. 1c) at 171, 193, 211 and $304 \AA$. They are obtained from running ratio difference images relative to the images taken $24 \mathrm{~s}$ earlier. The stack plots taken from the original images of these channels are shown in Supplementary Fig. S2. The white arrow indicates the signature of piled-up loops. b, The same as in a but for curve C2 at $131 \AA$. c, Flare light curves in RHESSI X-ray counts (3-6, 6-12 and 12-25 keV bands, left axis, colour-coded curves) and GOES X-ray fluxes (1-8 $\AA$, right axis, black curves). d, Signatures of inflow (orange) and outflow (cyan) indicated in the stack plots shown above. The grey curve shows the time derivative (times $10^{10}$ ) of GOES 1-8 $\AA$ flux as a proxy of the flare heating rate ${ }^{30}$. The two dashed cyan lines show the locations of the two cusps with time. e, Solid colour curves and plus signs show the calculated plasma inflow (orange) and outflow (cyan) velocities (left scale) as a function of time. Plus signs indicate that only a single velocity value was obtained. The black solid line shows the estimated reconnection rate $\left(M_{A}=V_{\text {in }} / V_{A} \approx V_{\text {in }} / V_{\text {out }}\right)$ as a function of time. All plots start on 17 August 2011 at 04:00:00.

a

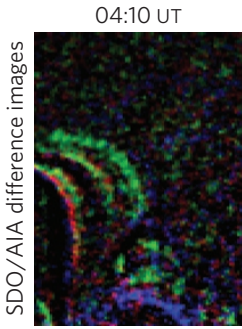

b

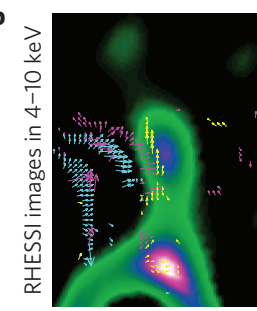

04:15 UT
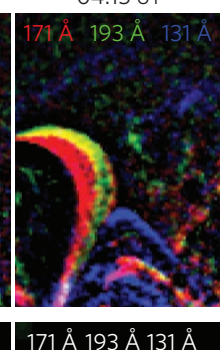

04:16 UT
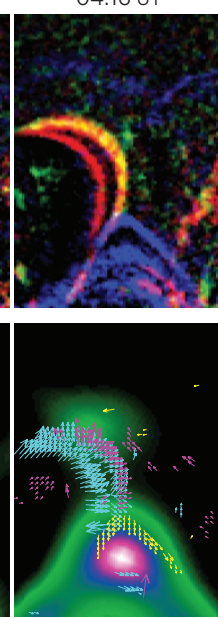

04:19 UT
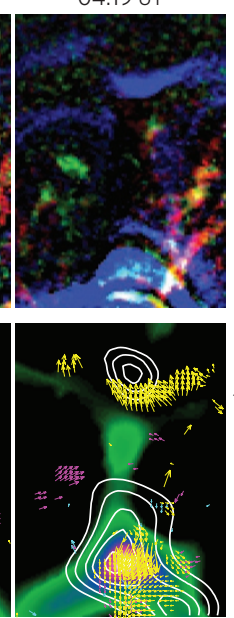

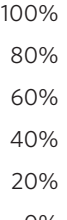

Figure 4 | Plasma flows and plasma heating in the reconnection region. This figure shows the curved, expanding inflow loops and the heated plasma observed in X-rays. It supports the idea that plasma in the outflow regions is heated during flux retraction. The area covered by these plots is indicated by the white rectangle in Fig. 1a and is rotated by $114^{\circ}$ to match Fig. 2. a, Red, green and blue represent enhanced emission in the AIA 171, 193 and $131 \AA$ images, respectively, relative to images taken 48 s earlier. b. RHESSI $4-10$ keV X-ray images with the colour code shown in the lower right expressed as the percentage of the peak intensity in each image. White contours in the fourth image show $10-20$ keV X-ray intensity at $10,20,40,60$ and $90 \%$ of the peak value. The coloured arrows indicate the flow velocity vectors from 10 to $80 \mathrm{~km} \mathrm{~s}^{-1}$ derived using the Fourier local correlation tracking ${ }^{26}$ method and a pair of AIA images taken $24 \mathrm{~s}$ apart in each of the same three channels. See also Supplementary Movie 5. 
arcade reconnect with each other. This inflow ${ }^{13}$ and the lateral development of the flare arcade are intrinsically three-dimensional phenomena ${ }^{28}$. Realistic three-dimensional simulations are required to understand the coronal magnetic reconnection and energy release processes observed here.

Received 19 December 2012; accepted 28 May 2013; published online 14 July 2013

\section{References}

1. Priest, E. \& Forbes, T. Magnetic Reconnection (Cambridge Univ. Press, 2000)

2. Yamada, M., Kulsrud, R. \& Ji, H. T. Magnetic reconnection. Rev. Mod. Phys. 82, 603-664 (2010).

3. Zhong, J. Y. et al. Modelling loop-top X-ray source and reconnection outflows in solar flares with intense lasers. Nature Phys. 6, 984-987 (2010).

4. Deng, X. H. \& Matsumoto, H. Rapid magnetic reconnection in the Earth's magnetosphere mediated by whistler waves. Nature 410, 557-560 (2001)

5. Øieroset, M., Phan, T. D., Fujimoto, M., Lin, R. P. \& Lepping, R. P. In situ detection of collisionless reconnection in the Earth's magnetotail. Nature 412, 414-417 (2001).

6. Aschwanden, M. J. Physics of the Solar Corona. An Introduction (Praxis, 2004).

7. Fletcher, L. et al. An observational overview of solar flares. Space Sci. Rev. 159, 19-106 (2011)

8. Shibata, K. \& Magara, T. Solar flares: Magnetohydrodynamic processes. Living Rev. Solar Phys. 8, 6 (2011).

9. Chen, P. F. Coronal mass ejections: Models and their observational basis. Living Rev. Solar Phys. 8, 1 (2011).

10. Yokoyama, T., Akita, K., Morimoto, T., Inoue, K. \& Newmark, J. Clear evidence of reconnection inflow of a solar flare. Astrophys. J. 546, L69-L72 (2001).

11. Li, L. P. \& Zhang, J. Observations of the magnetic reconnection signature of an M2 Flare on 2000 March 23. Astrophys. J. 703, 877-882 (2009).

12. Wang, T. J., Sui, L. H. \& Qiu, J. Direct observation of high-speed plasma outflows produced by magnetic reconnection in solar impulsive events. Astrophys. J. 661, L207-L210 (2007).

13. Savage, S. L. et al. Low-altitude reconnection inflow-outflow observations during a 2010 November 3 solar eruption. Astrophys. J. 754, 13-26 (2012).

14. Takasao, S., Asai, A., Isobe, H. \& Shibata, K. Simultaneous observation of reconnection inflow and outflow associated with the 2010 August 18 solar flare. Astrophys. J. 745, L6 (2012).

15. Tsuneta, S. et al. Observation of a solar flare at the limb with the YOHKOH Soft X-ray Telescope. Publ. Astron. Soc. Jpn 44, L63-L69 (1992).

16. Sui, L. H. \& Holman, G. D. Evidence for the formation of a large-scale current sheet in a solar flare. Astrophys. J. 596, L251-L254 (2003).

17. Lin, J. et al. Direct observations of the magnetic reconnection site of an eruption on 2003 November 18. Astrophys. J. 622, 1251-1264 (2005).

18. Liu, R. et al. A Reconnecting current sheet imaged in a solar flare. Astrophys. J. 723, L28-L33 (2010).

19. Masuda, S., Kosugi, T., Hara, H., Tsuneta, S. \& Ogawara, Y. A. loop-top hard $\mathrm{X}$-ray source in a compact solar flare as evidence for magnetic reconnection. Nature 371, 495-497 (1994).

20. Shibata, K. et al. Observations of X-ray jets with the YOHKOH soft X-ray telescope. Publ. Astron. Soc. Jpn. 44, 173-179 (1992).
21. Pesnell, W. D., Thompson, B. J. \& Chamberlin, P. C. The solar dynamics observatory (SDO). Sol. Phys. 275, 3-15 (2012).

22. Lemen, J. R. et al. The atmospheric imaging assembly (AIA) on the solar dynamics observatory (SDO). Sol. Phys. 275, 17-40 (2012).

23. Lin, R. P. et al. The Reuven Ramaty High-Energy Solar Spectroscopic Imager (RHESSI). Sol. Phys. 210, 3-32 (2002).

24. Liu, W., Wang, T. J., Dennis, B. R. \& Holman, G. D. Episodic X-Ray emission accompanying the activation of an eruptive prominence: Evidence of episodic magnetic reconnection. Astrophys. J. 698, 632-640 (2009).

25. Longcope, D. W. \& Guidoni, S. E. A model for the origin of high density in looptop X-ray sources. Astrophys. J. 740, 73-90 (2011).

26. Fisher, G. H. \& Welsch, B. T. in Subsurface and Atmospheric Influences on Solar Activity, ASP Conference Series Vol. 383 (eds Howe, R., Komm, R. W. Balasubramaniam, K. S. \& Petrie, G. J. D.) 373 (Astronomical Society of the Pacific, 2008).

27. Petschek, H. E. in The Physics of Solar Flares, NASA SP-50 (ed. Hess, W. N.) 425 (NASA, 1964).

28. Priest, E. R. \& Forbes, T. G. The magnetic nature of solar flares. Astron. Astrophys. Rev. 10, 313-377 (2002).

29. Schmahl, E. J., Pernak, R. L., Hurford, G. J., Lee, J. \& Bong, S. Analysis of RHESSI flares using a radio astronomical technique. Sol. Phys. 240, 241-252 (2007).

30. Neupert, W. M. comparison of solar $\mathrm{x}$-ray line emission with microwave emission during flares. Astrophys. J. 153, L59 (1968).

\section{Acknowledgements}

The authors dedicate this paper to the late RHESSI PI, R. P. Lin, in acknowledgement of his inspirational efforts that made possible the high-quality solar X-ray data used in this paper. RHESSI is a NASA Small Explorer Mission. The GOES is a joint effort of NASA and the National Oceanic and Atmospheric Administration (NOAA). The SDO is a mission for NASA's Living With a Star (LWS) Program. The work of Y.S. and A.M.V. was supported by the European Community Framework Programme 7, High Energy Solar Physics data in Europe (HESPE), grant agreement No. 263086. Y.S. also acknowledges NSFC 11233008 . The work of G.H. was supported by a NASA Guest Investigator Grant and the RHESSI programme. The work of T.W. was supported by NASA grant NNX12AB34G and NASA Cooperative Agreement NNG11PL10A to C.U.A. M.T. acknowledges the Austrian Science Fund (FWF): V195-N16. W.G. acknowledges 2011 CB811402 and NSFC 11233008.

\section{Author contributions}

Y.S. analysed the data, wrote the text and led the discussion. A.M.V., G.D.H., B.R.D., T.W., M.T. and W.G. contributed to the interpretation of the data and helped to improve the manuscript.

\section{Additional information}

Supplementary information is available in the online version of the paper. Reprints and permissions information is available online at www.nature.com/reprints. Correspondence and requests for materials should be addressed to Y.S.

\section{Competing financial interests}

The authors declare no competing financial interests. 